
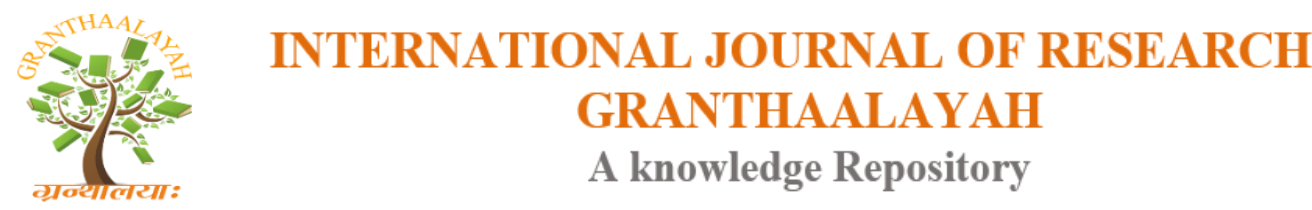

Science

\title{
DWT - SVD BASED MULTIPLE IMAGE WATERMARKING PROCESSES ON CLOUD COMPUTING
}

\author{
C. Ananth ${ }^{1}$, M. Karthikeyan ${ }^{2}$, N. Mohananthini ${ }^{3}$ \\ ${ }^{1,2}$ Assistant Professor / Programmer, Department of Computer and Information Science \\ Annamalai University, Tamilnadu, India \\ ${ }^{3}$ Assistant Professor, Department of Electrical and Electronics Engineering Muthayammal \\ Engineering College, Tamilnadu, India
}

\begin{abstract}
In the present work, the multiple watermarking techniques have been executed to present information security. DWT - SVD based multiple watermarking for color images are proposed and has been implemented in cloud computing. The multiple watermarking is used to share the copyright of multiple users improves the robustness and high security. In the proposed work, multiple watermarks are embedded into the cover image. Image quality is computed using Normalized Correlation (NC) and Peak Signal to Noise Ratio (PSNR) for color images. The proposed scheme provides a secure and well imperceptibility to different image processing attacks.

Keywords: Discrete Wavelet Transform; Singular Value Decomposition; Multiple Watermarking; Imperceptibility; Robustness.

Cite This Article: C. Ananth, M. Karthikeyan, and N. Mohananthini. (2018). "DWT - SVD BASED MULTIPLE IMAGE WATERMARKING PROCESSES ON CLOUD COMPUTING." International Journal of Research - Granthaalayah, 6(6), 88-96. https://doi.org/10.29121/granthaalayah.v6.i6.2018.1339.
\end{abstract}

\section{Introduction}

The recent advancements in digital information have made subtle changes in our society and life. The advantages of digital information also produced new challenges and opportunities. Innovations, supported by powerful software, new devices such as digital camera, camcorder and digital voice recorder, have reached the consumers, worldwide, use manipulate and experience the pleasure in the multimedia data. Internet and wireless communication networks give ubiquitous channels to send and exchange information.

Sumit Kumar Srivastava and HarikeshPandey [1] discussed the medical image watermarking techniques for protecting and authenticating the medical data. Their examine work studied the different hiding techniques and to perform an algorithm for watermarking the medical information of patients in medical images like MRI scan, X-Ray scan of medical images. Giakuomaki et al. 
[2] proposed a wavelet-based watermarking algorithm to embed multiple watermarks into medical images. Their method presented medical confidentiality and record integrity, the visual image quality of watermarked images which can be enhanced to achieve better PSNR values.

Sengul Doganet al. [3] proposed a watermarking System based on Discrete Cosines Transform (DCT), which is used to protect the security and integrity of transmitting biometric color images. Discrete wavelet transform based watermarking scheme is used for medical image watermarking using MATLAB software in [4]. Their proposed method provides good performance metrics and the medical image watermark technique can avoid unnecessary modification by unauthorized person. Mehta et al. [5] studied the performance of DWT, SVD and DWT-SVD based watermarking algorithms. In their method, two different watermarks are created; one is QR Code and the other one is a normal text image watermark. The result shows their DWT and SVD based methods are suitable for robustness. A multiple watermarking scheme based on discrete wavelet transform is presented for the analysis of imperceptibility and robustness in [6]. The watermarks are embedded into the detail sub-bands of original image using genetic algorithms. Their optimization to improve the performance of imperceptibility on the watermarked image in terms of peak signal to noise ratio (PSNR) and robustness for extracting watermark in term normalized correlation (NC).

Multiple watermarking techniques based on Hybrid DWT-SVD and Artificial Neural Network is proposed in [7]. Their proposed multiple watermarking technique, use of Artificial Bee Colony Algorithm based on DWT-SVD and artificial neural network. The understanding between the imperceptibility and robustness is considered as an optimization problem and is removed by applying an artificial bee colony algorithm.

Kavita Athnikar et al. [8] proposed a watermarking method in the architecture of cloud computing. It is designed for the purpose of mitigating the risk of insider disclosures. Their design represents all the requirements of the cloud oriented architecture framework. Shakun Gupta et al. [9] presented a watermarking technique has been implemented to provide data security. To implement the method of watermarking SVD-DCT-DWT techniques has been executed on cloud architecture. The robustness of the watermarked data, sharpened, contrast and salt-pepper attacks has been generated and presentation is analyzed with PSNR and MSE value. In their proposed technique, KALMAN filter is executed with SVD-DCT-DWT technique to get better PSNR and MSE value of the extracted image in watermarking.

Chao-Tung Yang et al. [10] proposed a method that can process image watermarking based on a robust method which combines the Singular Value Decomposition (SVD) and Distributed Discrete Wavelet Transformation (DDWT) over cloud computing environments. The Hardtop system with the integrate functions, HDFS and Map Reduce will play the key roles in this implementation. Boopathy D and M.Sundaresan [11] represented the encryption method for public cloud and also the cloud service provider's verification mechanism using the third party auditors with framework model. The Cloud Data Storage is one of the mandatory services which are acquired in this rapid development business world. The literature covering DWT based image watermarking scheme is found to be less robust for images. Animal Joseph et al. [12] proposed a robust watermarking algorithm based on DWT and SVD. 
In their proposed algorithm, the embedding of one watermark into HL band and another watermark embedded into the LH band of the host image and the robustness of extracted watermark is found to have low $\mathrm{NC}$ values for test image. In the present multiple watermarking techniques, two watermarks are embedded into LL2 sub bands of original image using discrete wavelet transform and singular value decomposition which in turn achieves more robustness. In an earlier work [13], the robustness of extracted watermark were found to be low, whereas in the present DWT and SVD based multiple watermarking techniques achieves more robustness.

\section{Preliminaries}

The proposed work focuses on multiple watermarking technique using DWT and SVD. The proposed methodology are discussed as follows,

\subsection{Composite Watermarking}

Image fusion is the process of mixing information from two or more images of a scene in to a single composite image that is certainly more beneficial and is also far better with regard to visible conception or computer finalizing. The objective of image fusion is to combine supporting multisensor, multi-temporal and multi-view facts directly into one particular new image. The aim should be to decrease uncertainty and limit redundancy in the productivity while maximizing applicable facts certain with a software or even process. Consider if the composite watermark image size is $200 * 200$; the 4 images to be watermarked are taken as $50 * 50$ which will be equal to $200 * 200$. Among the RGB values in the medical images, $R$ value is taken into account the remaining $G$ and $\mathrm{B}$ values were added. Hence the image to be watermarked is made by means of composite watermarking 4 images. Then the watermarking process is carried out.

\subsection{Hybrid Method}

The heart of wavelet analysis is a multi-resolution analysis (MRA). Multi resolution analysis is the decomposition of an image into different sub images of different size resolution levels. MRA is designed at high frequencies, to provide poor frequency resolution and good time resolution. MRA is designed at low frequencies, poor time, frequency resolution and good frequency resolution. The SVD is a powerful matrix decomposition tool which has also been used in a variety of applications. From the viewpoint of linear algebra, a discrete image can be represented as a 2D matrix with nonnegative scalar entries.

\subsection{Cloud Computing}

In the cloud computing technology shared information assets and software that is provided with computers and various other devices on demand. Electronic mail (e-mail) was maybe first service happening the "cloud". By utilizing the cloud technology, the peers can be entirely released from the troublesome local data storage and preservation. However, this technology also poses an important threat to the confidentiality of those stored information. With the assist of Cloud Computing, peers can access their stored information from anywhere on the planet only if they linked to the internet. Nowadays world depends on cloud computing to store their public information as well as personal information. That information may be necessary to them or others 
at any moment of time. As a result, information security in cloud computing has required lots of concentration from the research society. Hence the proposed work to concentrate the cloud security using the multiple watermarking technology.

\section{Proposed Method}

In cloud computing technology, which carries many benefits when occurs as a platform of information and resource sharing. It also hosts main security concerns. In our proposed work the technique of multiple watermarking had been applied in cloud computing. The multiple watermarking techniques had provided additional security to cloud data. The proposed work focuses on cloud computing using DWT, SVD and composite watermarking technique. The proposed methodology is discussed as follows,

The block diagrams of embedding and extraction method are shown in Figure 1 and 2.

\subsection{Watermark Embedding Method}

1) Mixing the information of first and second watermark image into a composite watermark image.

$\mathrm{W} 12=[\mathrm{W} 1 \mathrm{~W} 2 \mathrm{~W} 3]$

2) The original image (I) is decomposed into two levels using discrete wavelet transform.

3) The SVD process is applied to LL2 sub band of the original image

$$
I=U S V^{T}
$$

Alike, the SVD process is functional to composite watermark image

$$
W_{12}=U_{12} S_{12} V_{12}^{T}
$$

The singular value of composite watermark is embed in the singular value of the original image

$$
S_{W I}=S+\alpha S_{12}
$$

Here $\alpha$ is the scaling factor which determine the strength of the watermark.

The inverse SVD process is applied

$$
I_{W I}=U S_{W I} V^{T}
$$


The inverse wavelet transform is performed to get watermarked image

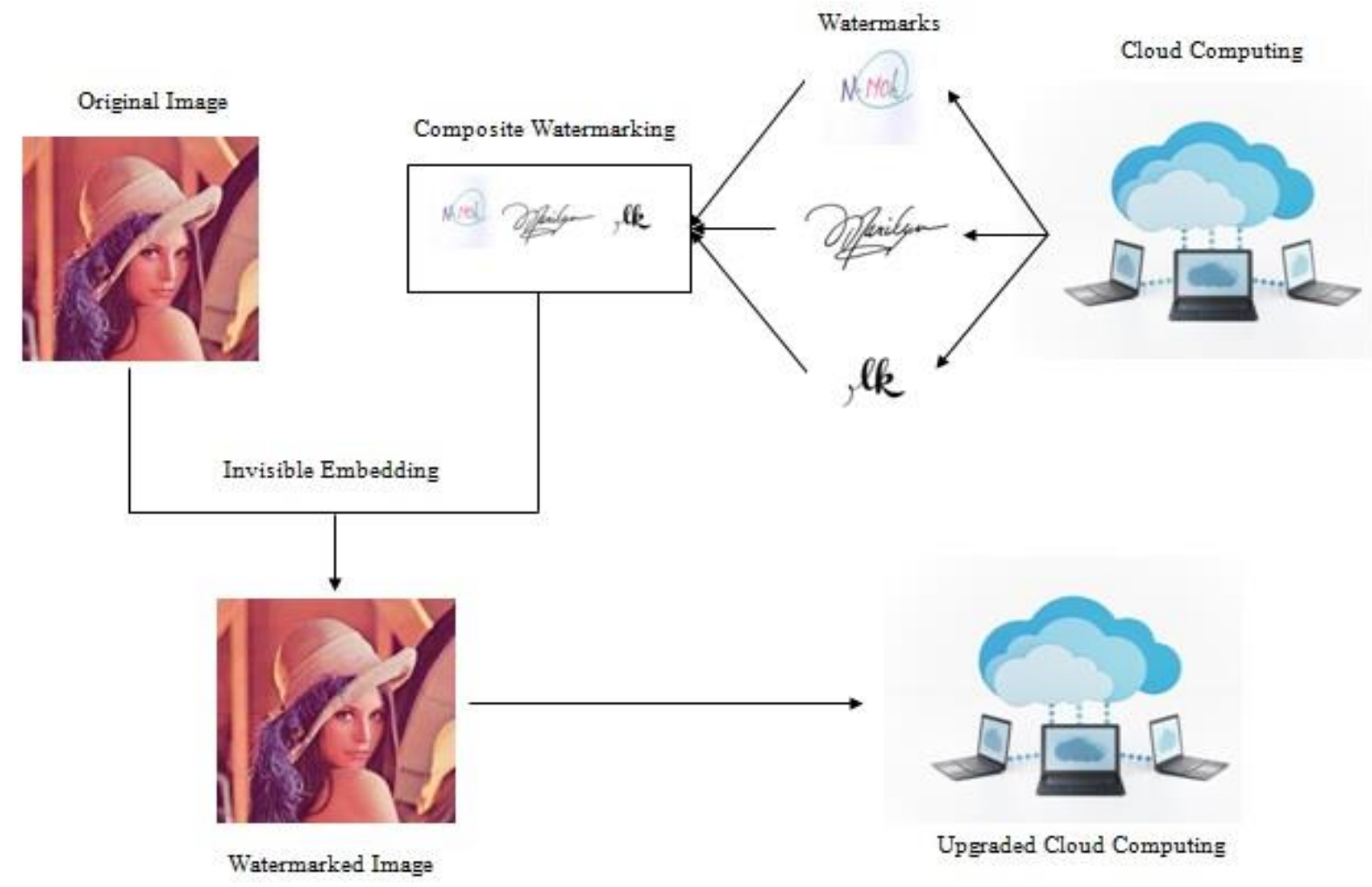

Figure1: The block diagram of embedding method

\subsection{Watermark Extraction Method}

1) The watermarked image and original image are decomposed into two levels using discrete wavelet transform.

2) The SVD process is applied to LL2 sub bands of watermarked image and original image

$$
I_{W I}=U S_{W I} V^{T}
$$

$I=U S V^{T}$

The singular values of composite watermark can be extracted as

$$
S_{12}^{\prime}=\left(S_{W I}-S\right) / \alpha
$$

The inverse SVD process is applied to composite watermark.

$W_{12}=U_{12} S_{12}^{\prime} V_{12}^{T}$ 
The first, second and third watermark images are obtained from the composite watermark image.

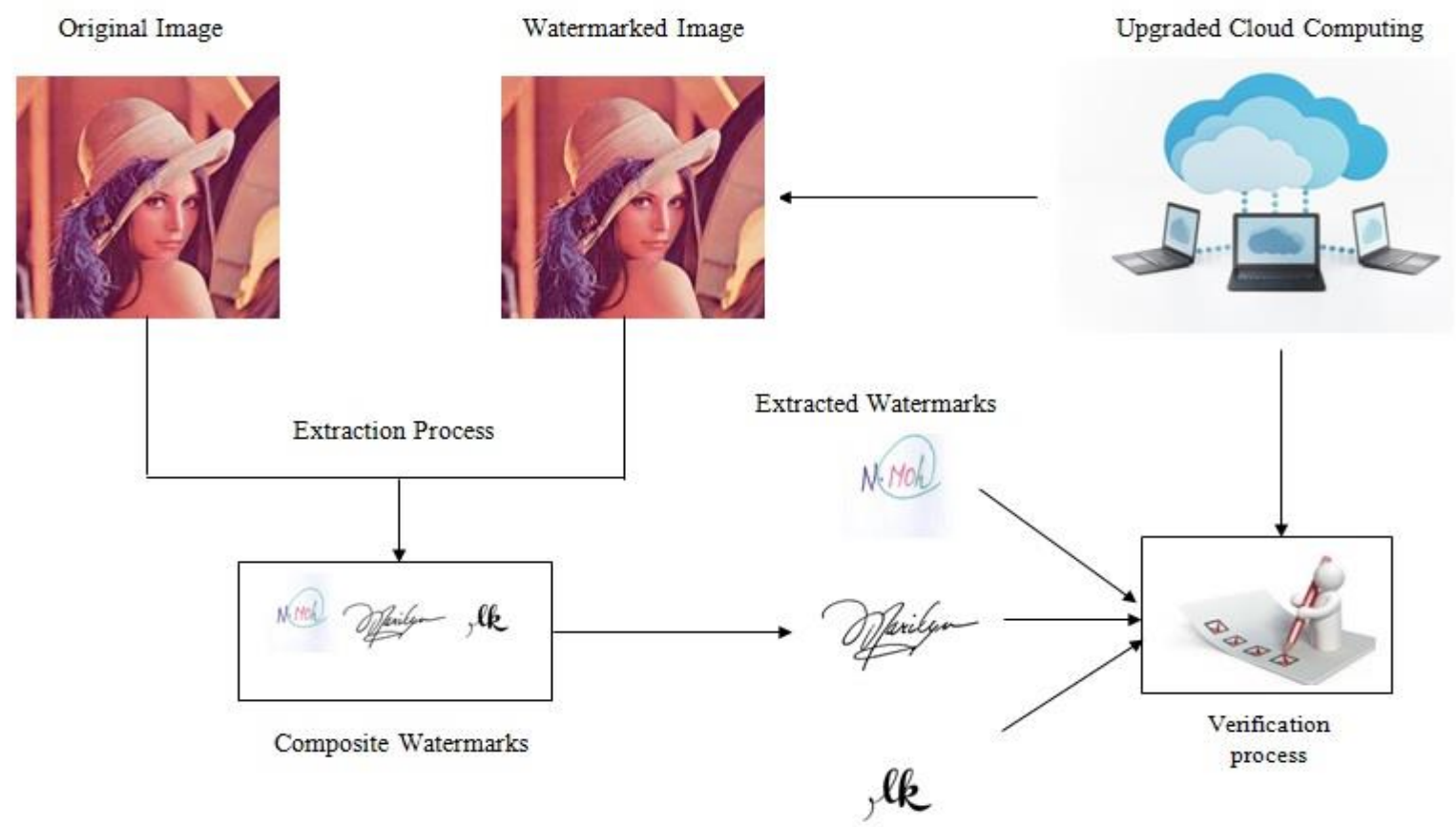

Figure2: The block diagram of extraction method

\section{Results and Discussion}

In this paper, a robust multiple watermarking technique is proposed based on wavelet domain for Color images. Figure 3 shows the original image of size $512 \times 512$ and the image watermarks of size $48 \times 48$. Peak Signal to Noise Ratio (PSNR) is the logarithmic value of ratio between signal and noise. It is expressed in decibels. The PSNR is used to measure quality of watermarked image as follows:

$\operatorname{PSNR}(d B)=10 \log _{10} \frac{255^{2}}{M S E}$

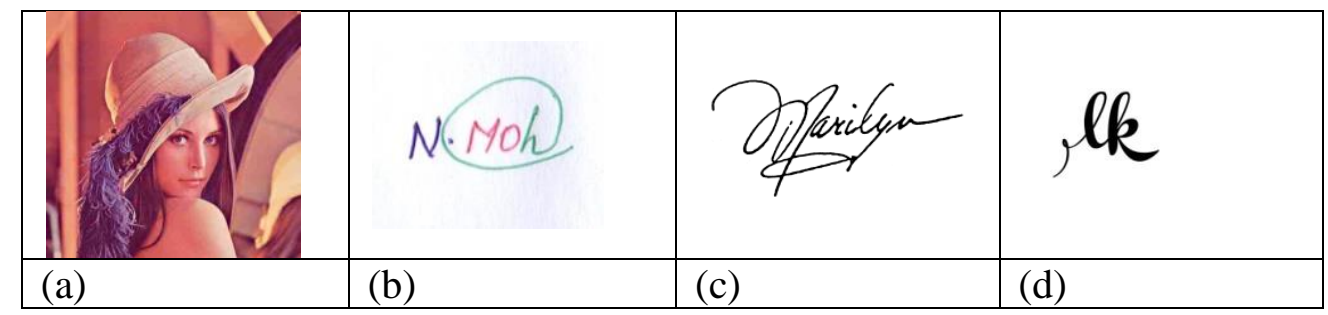

Figure3: Original image and Watermark Images

Where, $\mathrm{MSE}=$ Mean square error

Normalized Correlation (NC) is used to measure the quality of the watermark after recovery. The $\mathrm{NC}$ between the embedded watermark $\mathrm{W}(\mathrm{i}, \mathrm{j})$ and the extracted watermark $\mathrm{W}^{\prime}(\mathrm{i}, \mathrm{j})$ is given by 


$$
N C=\frac{\sum_{i=1}^{H} \sum_{j=1}^{L} W(i, j) \times W^{\prime}(i, j)}{\sum_{i=1}^{H} \sum_{j=1}^{L}[W(i, j)]^{2}}
$$

Table 1 show the PSNR and NC values on composite watermarking technique without and with attacks for Lena image. To prove the robustness, the watermarked images are tested with selected attacks such as geometric attacks, removal attacks and common image processing attacks on multiple watermarking techniques.

Table1: PSNR Values on Composite Watermarking Techniques against Attacks

\begin{tabular}{|l|l|l|l|l|}
\hline Attacks & Watermarked Image & \multicolumn{4}{|l|}{ Extracted Watermark } \\
\hline & PSNR (dB) & $\mathbf{1}$ & $\mathbf{2}$ & $\mathbf{3}$ \\
\hline Without Attacks & 43.8860 & 1 & 1 & 1 \\
\hline Salt \& Pepper Noise & 20.3567 & 0.82912 & 0.7959 & 0.8959 \\
\hline Gaussian Noise & 20.2297 & 0.8463 & 0.8303 & 0.7305 \\
\hline Speckle Noise & 27.9312 & 0.9991 & 0.9992 & 0.9992 \\
\hline Median Filtering & 34.8643 & 0.9986 & 1 & 1 \\
\hline Wiener Filtering & 20.5709 & 0.7065 & 0.7472 & 0.7810 \\
\hline Translation & 16.7612 & 0.5971 & 0.6366 & 0.6216 \\
\hline Cropping & 19.0532 & 0.3616 & 0.3247 & 0.3398 \\
\hline Rotation & 9.8369 & 0.1238 & 0.1889 & 0.1763 \\
\hline JPEG Compression & 36.3725 & 1 & 1 & 1 \\
\hline Sharpening & 25.5712 & 0.9332 & 0.9644 & 0.9492 \\
\hline Smoothing & 40.9808 & 1 & 1 & 1 \\
\hline Row-Column Blanking & 15.0812 & 0.0187 & 0.0743 & 0.0513 \\
\hline Row-Column Copying & 18.7832 & 0.9432 & 0.9042 & 0.9743 \\
\hline
\end{tabular}

The watermarked images are corrupted with salt and pepper noise at the density of 3\%, Gaussian noise of variance $1 \%$ and speckle noise of variance 0.005 . For median and wiener filtering are $3 \times 3$ mask consisting of 0.03 intensity values is used to reduce noise in the image. In cropping attack, a little piece of the watermarked image is slash or removed. Rotation is tested by rotating the image in 60 degrees direction and then back to the original position through bilinear interpolation. The watermarked images are compressed with quality factor 20. Sharpening operations are used to enhance the subjective quality. In row-column blanking attack, a set of rows and columns are deleted from 150 to 170 and 350 to 370. In row-column copy attack, a set of rows and columns are copied to the adjacent or random locations. In this attack from 151 to 200 rows are copied to 251 to 300 and 251 to 300 rows are copied to 151 to 200 . Table 1 shows the NC values on composite watermarking technique for the Lena color image. Commencing the results, it is obvious that, composite watermarking technique achieves more robustness for sharpening, speckle noise, JPEG compression, median filtering, sharpening, row-column copying and smoothing attacks watermarking achieves high PSNR values. 


\section{Performance Comparison to Existing Method}

To prove the effectiveness of the composite watermarking technique, the NC value is compared with the DWT - SVD based multiple watermarking methods [12]. Their method, one watermark is embedded to HL band and another watermark is embedded to a LH band of the original image. In the current multiple watermarking techniques, the multiple watermarks are embedded into LL sub bands of the original image.

Table2: Comparison to Existing Method

\begin{tabular}{|c|c|c|c|c|c|c|}
\hline \multirow[t]{2}{*}{ Attacks } & \multicolumn{2}{|c|}{ Existing Method [12] } & \multirow{2}{*}{$\begin{array}{c}\text { Existing Method [13] } \\
-\end{array}$} & \multicolumn{3}{|c|}{ Proposed Method } \\
\hline & NC1 & NC2 & & NC1 & NC2 & NC3 \\
\hline Gaussian Blur & 0.9869 & 0.9893 & - & 0.9875 & 0.9932 & 0.9812 \\
\hline Gaussian Noise & 0.7257 & 0.7371 & 0.417 & 0.8463 & 0.8303 & 0.7305 \\
\hline $\begin{array}{lll}\text { Salt \& Pepper } \\
\text { Noise }\end{array}$ & 0.8961 & 0.9191 & 0.400 & 0.82912 & 0.7959 & 0.8959 \\
\hline Speckle Noise & 0.9151 & 0.9347 & - & 0.9991 & 0.9992 & 0.9992 \\
\hline Cropping & - & - & 0.273 & 0.3616 & 0.3247 & 0.3398 \\
\hline Sharpening & - & - & 0.263 & 0.9332 & 0.9644 & 0.9492 \\
\hline Median Filtering & - & - & 0.612 & 0.9986 & 1 & 1 \\
\hline
\end{tabular}

The NC values are listed in Table 2 and it is evident that the robustness of performance of the proposed is superior to the existing method for the Lena image. To prove the effectiveness of the proposed multiple watermarking techniques, the $\mathrm{NC}$ value is compared with SVD-based watermarking method [13]. Their method, the watermarks are embedded in the singular values of DWT sub band into the original image.

\section{Conclusion}

The cloud computing architecture has been proposed in multiple watermarking technique with a certain number of peers. The peers can present their identification to the cloud services provider and cloud service provides access to peers. In this proposed work, the multiple watermarking technique has been executed to provide additional data security. Further, the experimental result shows that the DWT - SVD based Multiple image Watermarking processes based multiple watermarking achieves more imperceptibility and robustness.

\section{References}

[1] Sumit Kumar Srivastava, HarikeshPandey (2016), "MEDICAL IMAGE WATERMARKING WITH PATIENT DETAILS AS WATERMARK", International Journal of Advance Research, Ideas and Innovations in Technology, 2 (6), 1-6. https://www.ijariit.com/manuscript/medicalimage-watermarking-patient-details-water mar k/

[2] Giakoumaki A, Pavlopoulos S and Koutouris D, (2003) "A MEDICAL IMAGE WATERMARKING SCHEME BASED ON WAVELET TRANSFORM”, Proceedings of the 25th Annual International Conference of the IEEE Engineering in Medicine and Biology Society, 1, https://doi. 10.1109/iembs.2003.1279900.

[3] Sengul Dogan, Turker Tuncer, Engin Avci and Arif Gluten (2012), “A NEW WATERMARKING SYSTEM BASED ON DISCRETE COSINE TRANSFORM (DCT) IN COLOR BIOMETRIC 
IMAGES”, Journal of Medical Systems, 36, 2379-2385. https://doi.org/10.1007/s10916-0119705-2.

[4] C.Thirumaraiselvi, R.Sudhakar, G. Priyadharshini, G. Kalaivani (2016), "EMBEDDING PATIENT INFORMATION IN MEDICAL IMAGES USING WATERMARKING APPROACH FOR AUTHENTICATION", South Asian Journal of Engineering and Technology, 2 (18), 33-40. doi: 10.1109/ISCO.2016.7726895.

[5] Mehta, S. Nallusamy, R. Marawar, R.V. and Prabhakaran, B, (2013) "A STUDY OF DWT AND SVD BASED WATERMARKING ALGORITHMS FOR PATIENT PRIVACY IN MEDICAL IMAGES", IEEE International Conference on Healthcare Informatics (ICHI), 287 - 296. doi: 10.1109/ICHI.2013.41.

[6] N. Mohananthini and G. Yamuna, (2016) "PERFORMANCE OPTIMIZATION OF MULTIPLE WATERMARKING USING GENETIC ALGORITHMS", (2016), International Journal of Enterprise Network Management, Inderscience, 7 (3), 237- 249. https://doi.org/10.1504/IJENM.2016.078970.

[7] Mohananthini. N and Yamuna. G, (2015) "MULTIPLE IMAGE WATERMARKING TECHNIQUE BASED ON HYBRID DWT-SVD AND ARTIFICIAL NEURAL NETWORK", International Journal of Applied Engineering Research, 10 (3), 7275-7297.

[8] Kavita Athnikar, Prajakta Shirke, Snehal Tajave and Mukta Tarkasband, (2014) "A CLOUDBASED WATERMARKING METHOD FOR HEALTH DATA AND IMAGE SECURITY, International Journal of Computer Trends and Technology, 16 (3). DOI: 10.14445/22312803/IJCTT-V16P129.

[9] Shakun Gupta, Harsimran Singh, (2015) "TO PROPOSE A NOVEL TECHNIQUE FOR WATERMARKING IN CLOUD COMPUTING", International Journal of Engineering Development and Research, 3 (2).

[10] Chao-Tung Yang, Chu-Hsing Lin and Guey-Luen Chang (2011), "IMPLEMENTATION OF IMAGE WATERMARKING PROCESSES ON CLOUD COMPUTING ENVIRONMENTS", CCIS 223, 131-140.

[11] Boopathy D and M.Sundaresan, (2014) "DATA ENCRYPTION FRAMEWORK MODEL WITH WATERMARK SECURITY FOR DATA STORAGE IN PUBLIC CLOUD MODEL, International Conference on Computing for Sustainable Global Development (INDIACom), DOI: 10.1109/IndiaCom.2014.6828094.

[12] Anumol Joseph and Anusudha K, (2013) "ROBUST WATERMARKING BASED ON DWTSVD”, International Journal on Signal and Image Security, 1 (1), 01-05.

[13] Ghafoor A and Imran M, (2012) "A NON-BLIND COLOR IMAGE WATERMARKING SCHEME RESISTANT AGAINST GEOMETRIC ATTACKS”, Radio Engineering, 21 (4), 12461251.

*Corresponding author.

E-mail address: ananth.prog@gmail.com/ karthiaucse@gmail.com/ mohananthini@ yahoo.co.in 